\title{
Carnets
}

Revue électronique d'études françaises de l'APEF

Deuxième série - 3 | 2015

Insulaire

\section{Pour une géographie littéraire : une lecture d' Archipel de Claude Simon}

\author{
Michel Collot
}

\section{(2) OpenEdition}

Journals

Édition électronique

URL : http://journals.openedition.org/carnets/1380

DOI : 10.4000/carnets. 1380

ISSN : 1646-7698

Éditeur

APEF

Référence électronique

Michel Collot, «Pour une géographie littéraire : une lecture d'Archipel de Claude Simon », Carnets [En ligne], Deuxième série - 3 | 2015, mis en ligne le 28 février 2015, consulté le 19 avril 2019. URL : http:// journals.openedition.org/carnets/1380; DOI : 10.4000/carnets.1380

Ce document a été généré automatiquement le 19 avril 2019

\section{(c) (i) (8)}

Carnets est mis à disposition selon les termes de la licence Creative Commons - Atribution - Pas d'utilisation commerciale 4.0 International. 


\title{
Pour une géographie littéraire : une lecture d'Archipel de Claude Simon
}

\author{
Michel Collot
}

1 Depuis une vingtaine d'années au moins, un nombre important de travaux, ouvrages et colloques, ont été consacrés, dans plusieurs pays, à l'étude de l'inscription de la littérature dans l'espace et/ou à la représentation des lieux dans les textes littéraires. On peut se demander si l'on n'assiste pas à l'émergence d'une nouvelle discipline, qu'on pourrait appeler la "géographie littéraire» (Collot, 2014). Elle recouvre une grande diversité d'approches, que reflète la multiplicité des termes forgés pour les désigner: géopoétique, géocritique, geophilologie...; mais toutes supposent un lien entre la littérature et la géographie. Cette relation ne va pas de soi au regard des théories qui ont longtemps dominé les études littéraires françaises et qui proposaient d'analyser les textes en faisant abstraction de toute référence au réel et au monde extérieur.

2 Cette orientation récente de la critique et de la théorie littéraire est une réponse à l'évolution des pratiques d'écriture elles-mêmes qui font une place de plus en plus importante à des thèmes géographiques et à des formes spatialisées. Cette promotion de « la forme spatiale » a été repérée dès 1945 par le critique américain Joseph Frank dans un article célèbre (Frank, 1976). Elle concerne non seulement la poésie, qui explore de plus en plus librement l'espace de la page, mais aussi le récit contemporain, qui tend à devenir un « récit d'espace » (Certeau, $1990: 170)$, et par là-même souvent un "récit poétique » (Tadié, 1978) comme le montre, sous des formes et à des titres divers, l'évolution de la production d'un Butor ou d'un Gracq.

3 Cet intérêt des écrivains et des littéraires pour les questions d'espace s'inscrit dans un contexte intellectuel et culturel plus général. Il répond sans doute au souci croissant de nos sociétés envers les questions d'environnement, mais aussi au développement de pratiques artistiques liées au site (comme le Land Art), et à l'évolution des sciences de l'homme et de la société, qui se montrent depuis au moins un demi-siècle de plus en plus attentives à l'inscription des faits humains et sociaux dans l'espace. On a pu parler à ce propos d'un « tournant spatial » ou d'un « tournant géographique ». 

des Annales a proposé d'élargir l'échelle de l'enquête historique à de longues périodes et à de vastes aires géographiques. Fernand Braudel en est ainsi venu à proposer le terme de «géohistoire » pour baptiser l'étude des relations qu'une société entretient avec son cadre géographique à travers la longue durée (Braudel, 1997: 114). La "nouvelle Histoire » se montre particulièrement attentive au paysage comme reflet des mutations de la mentalité collective. Et la géographie de son côté intègre de plus en plus la dimension historique, en devenant géographie humaine, économique, sociale et culturelle, plus que géographie physique. C'est pourquoi les géographes dits « humanistes » se sont beaucoup intéressés à la littérature, en réaction contre une autre tendance de la discipline qui, à la faveur du perfectionnement des moyens techniques, mathématiques et informatiques mis à sa disposition, a tendance à privilégier une analyse objective et abstraite de l'espace géographique aux détriments de sa dimension humaine et sensible. trouvant dans la littérature la meilleure expression de la relation concrète, affective et symbolique qui unit l'homme aux lieux, et les littéraires se montrant de leur côté de plus en plus attentifs à l'espace où se déploie l'écriture.

6 Il n'en reste pas moins nécessaire de bien marquer la spécificité littéraire des œuvres et de leur approche, si l'on ne veut pas transformer la géographie littéraire en une simple annexe de la géographie culturelle.

7 La littérature est une forme spécifique de géo-graphie, une façon d'écrire le rapport à la terre qui met en crise le savoir géographique, une géo-critique ; au lieu de reproduire les données géographiques, elle les réinvente et les recrée : elle est une géo-poétique.

\section{Archipel}

8 Pour illustrer l'intérêt que peut représenter une approche géographique, géocritique et géopoétique des textes littéraires, j'ai choisi d'étudier un texte de Claude Simon consacré à un archipel qui existe bien sur les cartes de l'Europe mais qui est remanié par son imaginaire et mis en forme par son écriture.

9 Dans ce qu'il est convenu d'appeler le Nouveau roman, la crise de la narration, la déconstruction du personnage et de l'intrigue se fait souvent au profit de l'espace. Dans ce contexte, l'œuvre de Claude Simon a toujours été fortement liée aux lieux, à la géographie autant qu'à l'histoire: un colloque intitulé «Claude Simon géographe » s'est tenu en 2011 à Toulouse (Laurichesse, 2013). Sa conception de l'Histoire elle-même est une géohistoire, qui, comme celle de Fernand Braudel, valorise la longue durée, au point d'apparaître comme une histoire immobile ou du moins répétitive. On se rappelle l'épigraphe de L'Invitation, empruntée à Bismarck: "Le seul facteur permanent de l'Histoire, c'est la géographie » (Simon, 1987). Et réciproquement sa géographie est faite de lieux de mémoire, d'une mémoire collective et personnelle : Les Géorgiques, qui disent l'attachement de Simon à la terre, relèvent du roman historique (Simon, 1981).

10 Dans les romans, cette attention aux lieux se traduit par l'expansion de la description qui tend, si ce n'est à « enliser le récit » (Ricardou, 1973 : 128), du moins à l'égarer ; mais elle reste prise dans une logique narrative, si ce n'est fictionnelle. Dans Archipel et dans Nord, deux textes récemment redécouverts et publiés ensemble (Simon, 2009), la fiction 
disparaît quasiment au profit du documentaire. Si récit il y a, il s'agit d'une relation de voyage, voire d'un reportage. La trame narrative s'y réduit à un parcours de l'espace et laisse le premier plan à la description ou à l'évocation des lieux et des paysages.

11 Cette prépondérance de la thématique spatiale répond certes à la demande des revues qui ont accueilli ces textes brefs ${ }^{1}$; elle me semble pourtant liée aussi à l'accentuation d'une dimension poétique, qu'on trouve ailleurs chez Simon, mais qui s'accuse très nettement ici, notamment dans Archipel, où elle se traduit, entre autres, par une mise en page du texte qui est une mise en espace. Cette poéticité, sous-jacente à toute l'œuvre de Claude Simon, s'affirme ici parce que la description s'y émancipe du cadre de la narration et de la fiction.

Mais elle n'exclut nullement une très grande précision dans l'évocation de la topographie de cet archipel. Je proposerai donc trois séries de remarques sur ce texte, qui proposeront successivement une étude de son référent géographique, une lecture de son imaginaire et une analyse de son écriture, c'est-à-dire une géographie, une géocritique et une géopoétique. Mais je n'oublierai pas de le situer d'abord dans l'histoire littéraire, géographie et histoire littéraires ne s'excluant nullement à mes yeux mais étant plutôt complémentaires.

\section{Histoire littéraire}

13 Ce texte a été publié en 1973 au Danemark, au moment même de ce qu'on a pu considérer comme le tournant "textualiste» de la production simonienne. Telle est du moins la lecture qu'a tenté d'imposer en 1974 le colloque de Cerisy, dirigé par Jean Ricardou. Or il me semble échapper à une telle lecture et à la conception de la littérature qui la sous-tend et que Simon n'a jamais faite sienne, même s'il a pu paraître dans les années 1970 lui donner, si ce n'est des gages, du moins des arguments. Son écriture est en effet tournée davantage vers la réalité extérieure que vers elle-même, et elle fait la part belle aux référents, ce qui n'était pas très bien vu alors des avant-gardes littéraires françaises.

Cette dimension référentielle tient sans doute à la nature particulière de ce texte, qui est un récit de voyage, et à son lieu de publication : la revue danoise qui l'a publié et peutêtre commandé à Simon n'est pas une revue littéraire, plutôt une brochure touristique. Elle porte le nom même de l'archipel : Åland. Le texte est issu de notes prises sur place, qui ont pu être complétées et mises en ordre après-coup. On pourrait en conclure que cette publication, parue dans un lieu excentrique, occupe une place très marginale dans l'œuvre de Claude Simon. Il n'en est rien à mes yeux : ce petit texte, si tardivement publié en France, me semble avoir été plutôt en avance sur la suite de la production simonienne et sur l'évolution générale des pratiques littéraires, qui vont l'une et l'autre, à partir des années 1980, donner au récit de voyage et à l'autobiographie une importance croissante. Comme quoi les lieux de publication excentriques sont parfois plus favorables à l'innovation que ceux qui sont situés au centre, plus soumis à l'air du temps.

15 Archipel se présente un peu comme un "reportage », ancré dans la réalité des contrées visitées par l'écrivain-voyageur. Il en décrit très précisément la géographie, la topographie, la végétation, la géologie, l'habitat, sans oublier d'en rappeler l'histoire. Simon semble ici se référer principalement à la connaissance directe qu'il a pu prendre des lieux qu'il évoque, même s'il a sans doute fait appel à une documentation 
complémentaire, qui a pu lui être fournie par ses hôtes. Plus que sur des choses lues, il s'appuie sur des choses vues et entendues, objets d'une expérience sensible et vécue.

\section{Géographie}

L'archipel qui donne son titre au texte est celui d' Åland, constitué de 6500 îles et îlots situés entre la Finlande et la Suède. On peut suivre sur une carte le trajet que le visiteur a effectué pour le rejoindre en avion depuis Helsinki, survolant d'abord une côte très découpée, à l'intérieur de laquelle l'eau s'insinue par de multiples échancrures, puis une mer parsemée de « débris » de terre.

\section{Carte administrative d'Åland}

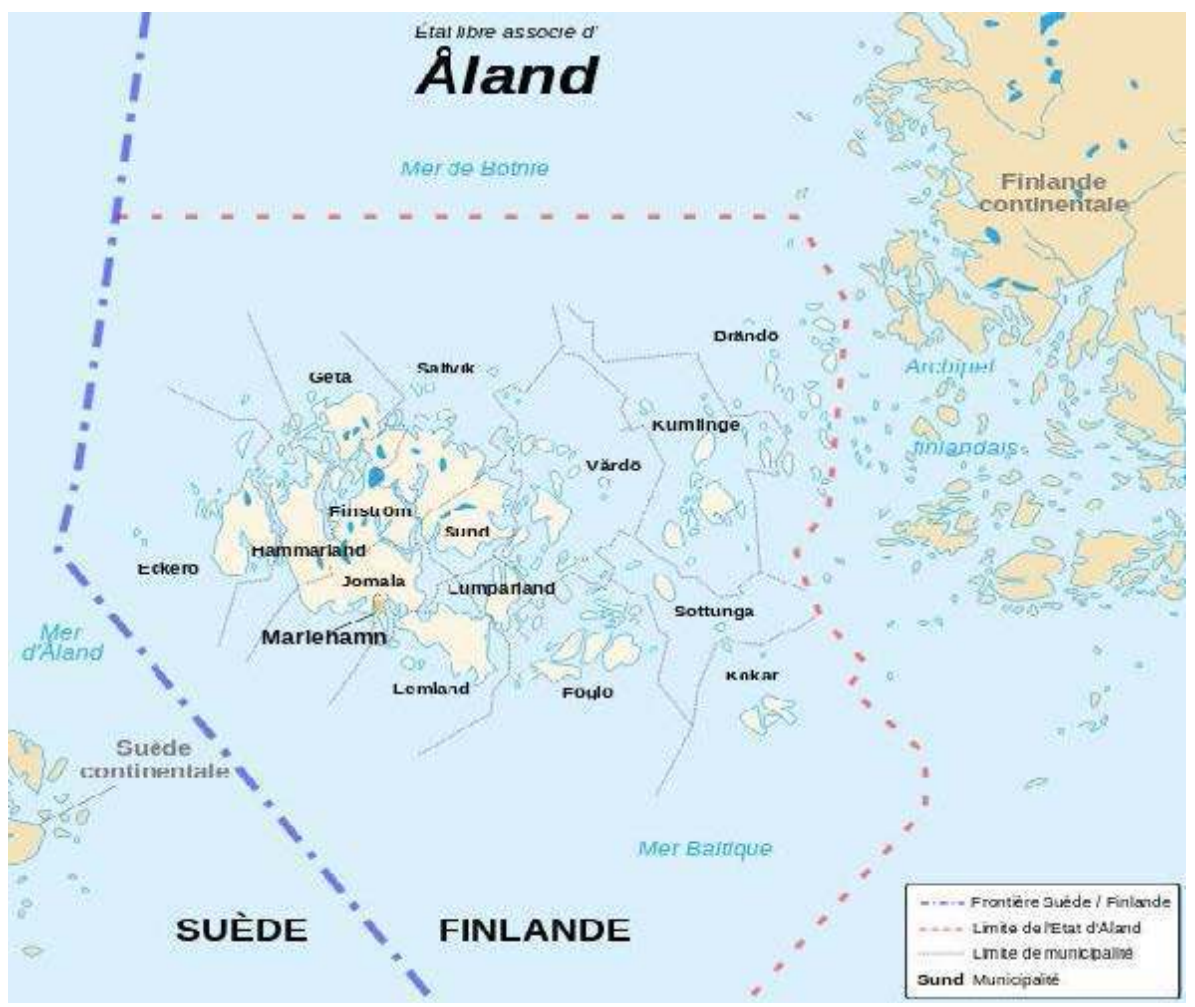

(C) Sémhur / Wikimedia Commons, via Wikimedia Commons

17 L'écrivain s'est intéressé à la géologie de cet archipel, constitué de granit rose érodé par les glaciations du Pléistocène ; il en évoque poétiquement la lente formation : «millions d'années aux épaisseurs bleuâtres rampant rabotant dans un formidable silence peuplé de formidables craquements le granit poli milliers d'îles milliers de golfes de baies de criques où s'arrondit la mer couleur d'huître » (Simon, 2009 : 11).

Ce travail de l'érosion glaciaire explique à la fois la disposition des îles «en longs chapelets parallèles » et leur relief caractéristique, le granit ayant été tellement aplani que certains îlots, "pas assez hauts pour qu'une seule vague ne les recouvre" ressemblent à des «poisson(s) pétrifié(s)» (Simon, 2009: 15) : «l'eau s'étalant en nappe sans se briser sur la surface polie du granit se retirant la laissant mouillée flanc d'une baleine lilas » (Simon, 2009 : 13). 
19 En bon observateur, Simon, guidé par ses accompagnateurs, remarque les stries laissées par le glacier au cours de son déplacement: "non pas roc mais cuir épais de vieux pachyderme sillonné de rides de fissures d'entailles entrecroisées laissées par un couteau ébréché » (Simon, 2009 : 13). À son regard informé n'échappent pas non plus les lichens qui recouvrent beaucoup de rochers, "gris-vert ou jaunes comme des pièces de monnaie mordant les unes sur les autres » (Simon, 2009: 15), ni les végétaux caractéristiques de la taïga : "touffes d'aulnes sorbiers frissonnant à peine et ces longues herbes comme des plumes roses formant de loin des nuages estompés pastel » (Simon, 2009 : 12-13).

Les notations concernant l'agriculture et l'habitat de l'archipel sont plus fugitives, et je n'y insisterai pas. Les allusions historiques sont en revanche nombreuses et insistantes, mais dispersées; elles ne s'organisent évidemment pas selon un ordre chronologique, mais interviennent à l'occasion de la visite de quelques lieux de mémoire. Ces derniers ne sont jamais nommés, mais on peut les identifier d'après les monuments qui y sont décrits ou les événements qui s'y sont déroulés. Ainsi, lorsque Simon relate une excursion en hydravion jusqu'à l'île de Kökar, il mêle à la description des vestiges du monastère l'évocation de l'arrivée des moines-soldats qui l'ont fondé au Xvi ${ }^{e}$ siècle :

Franciscains moines fanatiques déchaux venus d'où construire ici un sanctuaire de blocs roses lilas bistre cyclamen au toit couvert d'écailles peindre le flagellé le juge en robe prune qui se lave les mains sculpter ces grappes de sang coagulé (...)

casqués et armés de fer eux aussi sans doute ils avaient pris pied sur ces mêmes rochers débarquant de nacelles cloutées ceints de baudriers par-dessus leurs robes brunes (Simon, 2009 : p. 12-13).

21 Plus loin, c'est la visite de la forteresse de Bomarsund qui rappelle le siège qu'Anglais et Français lui ont fait subir pendant la guerre de Crimée, immortalisé par une "gravure " (Simon, 2009: 15-16) minutieusement décrite par Simon. Les conflits qui ont opposé pendant des siècles les « princesses » danoises et russes pour la possession de ces îles sont évoqués plus discrètement vers la fin du texte (Simon, 2009: 16), mais celui-ci se termine sur une extraordinaire énumération des différentes composantes de la topographie de l'archipel (« détroits », « isthmes » « îles », « lacs », « marécages », « criques ») avant de se résoudre dans « le blanc du silence » (Simon, $2009: 17)$.

La géographie l'emporte ici sur l'histoire, et la paix de la nature sur les violences des hommes; tout au long du texte, Simon oppose implicitement aux «fleurs» et au «tonnerre " « de feu » déchaînés par les envahisseurs la végétation paisible et le silence qui règnent désormais sur ce sanctuaire naturel :

nénuphars cernés de clair s'éparpillant sur le fond d'ardoise dérivant (...)

tonnerre soudain dans ces silences fleur de feu au cœur jaune aux pétales vermillon s'épanouissant combats pour ces détroits (Simon, 2009 : 11).

fleurs tonnantes de feu étraves bardées de fer (...)

comme de sacrilèges coups de tonnerre se répondant dans le silence répercutés par les glaces les roches le ciel vide (Simon, 2009 : 15).

Au terme d'une succession interminable de guerres, l'archipel d'Åland a été démilitarisé en 1922 ; bien que rattaché à la Finlande, il est devenu autonome et un traité garantit sa neutralité. Il est aujourd'hui à l'image de la Scandinavie telle que la présentait Simon dans son Discours de Stockholm : "une sorte d'îlot privilégié et exemplaire " "en bordure du monde de fer et de violence où nous vivons » (Simon, 2006 : 288). 

référentielle : étudier non plus le pays mais l'image du pays, c'est à dire le paysage, et non plus la carte. Cette notion que j'entends dans un sens proche de celui que lui a donné Jean-Pierre Richard (Collot, 2005 :177-189), me semble d'autant plus pertinente ici que ce texte, comme beaucoup d'autres de Simon, met l'accent sur le rôle de la perception en général, et du point de vue en particulier, dans l'élaboration de cette image. Il y a par exemple une sorte de réalisme phénoménologique dans l'évocation initiale de l'archipel vu d'avion, où le narrateur se montre attentif aux « effets d'optique » :

comme des accrocs d'abord, par places, comme si au-dessous du tissu de prés de bois de champs parallèles s'étendait un autre ciel, symétrique à celui où vole l'avion, plus foncé toutefois, d'un bleu légèrement violacé

ou gris

miroitant dans le contre-jour comme des glaces de métal à l'éclat terne enchâssées dans l'herbe

reflet citron parfois courant sur la surface quand le soleil

effet d'optique les sertissant de lumière comme si non pas trous mais ces flaques de mercure répandu faiblement en relief sur la terre assombrie (Simon, 2009 : 9).

La précision de ce regard de photographe n'exclut d'ailleurs ni le recours aux autres sens, à l'ouïe notamment, très sensible, on l'a noté, au silence des lieux, ni l'intervention de l'imaginaire : en même temps qu'il découvre ces îles, le voyageur « les imagin(e) peuplées de créatures fabuleuses » (Simon, $2009: 11)$.

Tout cela donne une forme et une signification bien particulières à ces deux archétypes géographiques que sont lîle et l'archipel. Ils procurent au voyageur un dépaysement total au sens le plus fort du terme : un bouleversement de sa vision du monde. Ce sont des terres inconnues, en marge de la civilisation: on pourrait interpréter en ce sens le toponyme Åland qui peut évoquer une sorte de bout du monde, éloigné de la civilisation. On sait l'attrait qu'exerce sur Simon cette sauvagerie qui délivre les lieux de l'emprise de la culture, voire de l'homme, et qui permet d'accéder à un réel dépouillé des représentations qui l'occultent habituellement. 
29 Au début d'Archipel, ce changement de point de vue est favorisé notamment par la perspective aérienne que permet le survol en avion et que Simon adopte souvent, parce qu'elle minimise les traces de la civilisation et de la présence humaine.

Elle brouille ici la distinction familière et fondamentale entre le ciel et la mer, le haut et le bas : tout se passe « comme si au-dessous du tissu de prés de bois de champs parallèles s'étendait un autre ciel, symétrique à celui où vole l'avion» (Simon, 2009: 9). Et au moment de l'atterrissage, « la mer l'archipel tout entier » semblent «mont(er) » vers les passagers de l'avion (Simon, 2009: 12). La topographie des côtes finlandaises et de l'archipel favorise en outre l'indistinction entre la terre et l'eau; les terres sont trouées de lacs, et la mer, parsemée d'îles, si bien que les rapports entre figure et fond, creux et pleins, sont perturbés voire inversés : miroitant dans la lumière, les étendues liquides qui s'infiltrent entre les terres apparaissent non pas comme des «trous», mais comme des "flaques de mercure répandu faiblement en relief sur la terre assombrie » (Simon, 2009: 9). Toute idée de continuité continentale et territoriale vole en éclats: la terre «se déchiqu(ète) se dépiaut(e) pour ainsi dire / haillon percé de mille déchirures »; et l'altitude fait perdre l'échelle des choses : la mer devient une «mare » (Simon, 2009: 10) et la campagne, miniaturisée, un « jardin » japonais (Simon, 2009 : 14).

31 Cette désorientation générale produit l'image d'un monde à l'envers. Le motif de l'inversion, familier aux lecteurs de Simon, revient comme un leitmotiv dans l'évocation de l'archipel :

la route obliquant vers le haut s'incurvant revenant sur la gauche en suivant la rive s'incurvant en sens inverse (...)

comme si l'avion survolait une de ces peintures un de ces jeux graphiques où de droite à gauche l'une des couleurs prend peu à peu la place de l'autre l'envahissant par fractions grandissantes chaque élément contraire en qualités égales au centre de la toile puis

l'inverse à présent : lambeaux s'étirant en longs chapelets parallèles (Simon, 2009 : 10).

Ce renversement culmine dans l'évocation de l'archipel des Cyclades qui se superpose à celui d'Åland, et du drapeau de la Grèce, image inversée de celui de la Finlande :

archipel ARXI-PELAGOS: primitivement non ces innombrables grains de terre semés mais au contraire la vaste mer

comme si le sens s'était inversé Contenant pour le contenu Grèce à l'envers (et de même les deux drapeaux l'un à croix blanche sur fond bleu l'autre à croix bleue sur fond blanc) Comme un positif photographique et son négatif sablier le haut en bas où le vide est plein langage retourné comme un gant les coutures ici devenant saillies (Simon, 2009 : 11).

Aux illusions d'optique s'ajoutent les équivoques sémantiques et symboliques. Le retour à un sens supposé premier du mot archipel, qui signifiait en grec ancien «la mer principale ", équivaut à une sorte de submersion linguistique des îles, tandis que le bleu et le blanc échangent leurs positions respectives dans les pavillons de la Grèce et de la Finlande. Si la littérature reflète le réel, c'est un reflet inversé comme sur un négatif photographique ou dans un miroir. En changeant le sens des mots, elle révèle l'envers de la réalité familière, que nous cachent nos habitudes de pensée et de langage. Ce motif du monde à l'envers est très présent dans l'œuvre de Simon; par exemple dans ce passage des Géorgiques, qui reprend la même image du gant retourné : «le monde à présent pour 
ainsi dire retourné à la façon d'un gant, d'un vêtement, révélé dans son envers ou plutôt perverti en ce sens que plus rien n'y avait la même signification, sinon de signification tout court » (Simon, $1981: 426)$.

Si un tel renversement est une épreuve, c'est aussi un révélateur. En inversant notre point de vue, littérature nous fait accéder à une vision plus lucide des choses. L'image chaotique et fragmentée qui nous est donnée de l'archipel déjoue les canons classiques de la description, mais elle correspond à une autre organisation du texte, fractale et fragmentaire. $\mathrm{Du}$ chaos procède un nouveau cosmos, peut-être un chaosmos, pour reprendre le mot-valise forgé par Joyce.

\section{Géopoétique}

Le sens de cette géographie revisitée par l'imaginaire simonien est indissociable de la forme qu'elle revêt dans ce texte et qui est selon moi l'objet propre d'une géopoétique ${ }^{2}$. Il y a dans ce texte une dimension poétique, qui est présente aussi dans les textes romanesques de Simon, mais qui est ici particulièrement saillante du fait de son émancipation vis à vis de tout cadre narratif et fictionnel. Je mettrai l'accent sur quelques-uns de ces traits de poéticité : la mise en valeur du signifiant, la typographie, la syntaxe, et la rhétorique.

La mention de mots et de noms étrangers permet la mise en valeur de leurs signifiants : ainsi la transcription en lettres grecques capitales du mot qui donne son titre au texte: ARXI-PELAGO (Simon, 2009: 11) produit un effet d'étrangeté et de dépaysement par l'emprunt à un autre alphabet qui rend pour certains lecteurs au moins l'inscription non seulement illisible mais imprononçable (il y a d'ailleurs une faute dans le texte original : il manque un sigma à la fin du mot). C'est un procédé fréquent dans l'oeuvre de Simon, qu'on trouve en particulier dans La Bataille de Pharsale, et qui met en valeur la dimension graphique, voire plastique des mots (Simon, 1969). L'écrivain se fait peintre non seulement quand il décrit les paysages mais quand il évoque les impératrices aux «noms bleus et blancs bleus et rouges Alexandra Kristina Katherina » (Simon, 2009 : 16).

La mise en page de ce texte le soumet à un travail de fragmentation et de spatialisation comparable à celui qu'on rencontre dans la poésie contemporaine, et en tout cas beaucoup plus poussé que dans les autres œuvres de Simon, où la continuité typographique masque le plus souvent les discontinuités sémantiques et syntaxiques. Les blancs et les alinéas viennent remplacer ici la ponctuation, presque totalement absente à la fin des séquences. Ils isolent notamment de façon spectaculaire certains syntagmes, qui, dès lors, se rapprochent visuellement, et parfois prosodiquement, du vers. Leur poéticité est d'autant plus marquée qu'il s'agit souvent de métaphores, et elle est renforcée par des recherches de rythme et de sonorité: "haillon percé de mille déchirures ", décasyllabe (Simon, 2009: 10); "clapotis de silence», hexasyllabe, allitération et assonance (Simon, $2009: 14$ ).

Beaucoup d'énoncés plus longs s'interrompent eux aussi avant la justification, sans attendre que la phrase et le sens soient complets, s'arrêtant parfois net sur un mot outil qui appelle une suite qui ne viendra pas, imitant l'effet produit en poésie par le rejet ou le contre-rejet :

colonnes processions de pélerins cheminant fantastique armada cinglant vers

(Simon, 2009: 10). 
Les paragraphes s'apparentent à des strophes, ponctuées parfois d'anaphores ou de leitmotive, telle la répétition obsédante du mot «silence», créant des parallélismes analogues à ceux de la poésie, particulièrement apparents dans la longue énumération qui conclut le texte :

princesses aux dots composées d'archipels aux couches remplies d'îles

de forêts grandes comme des continents

se disputant entre elles disputant aux usurpateurs venus du Sud

les détroits

les isthmes

les îles nénuphars

les lacs de mercure

les processions d'îles

les marécages

les caravanes d'îles sur la mer d'étain

les criques

les joncs pâles

les hommes poissons aux corps de neige aux arêtes roses aux femellles à tétines dessinées en rose saumon sur le blanc du silence (Simon, 2009 : 16-17).

Le texte se termine sur l'évocation de fresques d'allure fantastique peintes sur les murs blancs du monastère de Kökar. Cette série de brefs syntagmes alignés verticalement sur la page crée un rythme visuel inhabituel, où les blancs l'emportent sur les noirs, donnant à voir le rapport de l'écriture avec « le blanc du silence » qui est essentiel à la poésie. On a ici affaire à un véritable archipel typographique, l'espacement du texte correspondant à la dispersion des îles à la surface de la mer, nous donnant à lire cette «parole en archipel » qui est, selon Char, celle de la poésie.

41 Cette discontinuité typographique se double, dans Archipel, d'un véritable éclatement de la syntaxe. Ce texte ne comporte aucune phrase complète, du fait de l'usage systématique de la tournure nominale. Celle-ci est caractéristique du style de l'annotation. Tout se passe comme si Claude Simon avait délibérément renoncé à mettre ses notes en phrases et en phase les unes avec les autres. Mais la syntaxe nominale est aussi très présente dans la poésie contemporaine, qui cultive volontiers «le style substantif » (Meschonnic, 1972: 8), dressant le mot dans son superbe isolement comme « un signe debout " (Barthes, $1972: 39)$.

Dans les autres textes de Simon, elle est le plus souvent en général réservée aux passages descriptifs ; mais tout se passe comme si la description était dans Archipel généralisée, au point que la distinction et la hiérarchie entre narration et description se trouve effacée ou renversée ; en quoi ce texte apparemment marginal est peut-être la réalisation la plus radicale d'un idéal auquel Simon a souvent rêvé : «n'est-il pas possible de concevoir un 
système romanesque entièrement neuf, entièrement inversé, c'est-à-dire où la description deviendrait tout de bon l'élément principal» (Simon, 1980:87). Or on a pu montrer les affinités entre poésie et description, qui l'une et l'autre explorent davantage l'axe paradigmatique que l'axe syntagmatique du discours (Hamon, 1990 : 44-45).

La syntaxe nominale, c'est aussi le style de la "chose vue » : un énoncé tourné vers son objet qui fait l'économie du sujet de l'énonciation (Benvéniste, 1966 : 159). Et de fait, on ne trouve dans Archipel aucune occurrence du pronom de la première personne. S'agirait-il d'un exemple de "poésie objective", conforme au vœu de Rimbaud? L'absence de prédication me semble pouvoir être interprétée plutôt comme l'expression d'une expérience antéprédicative, antérieure au jugement, où le sujet ne se distingue pas du monde (Collot, 1997 : 282-285). Elle serait ici au service de cette écriture de la sensation que cultivent aussi bien Claude Simon que la poésie contemporaine (Castin, 1998; Zenmour, 2008).

Le " principe d'équivalence » qui, selon Jakobson, domine en poésie, régit fortement les textes de Simon, aussi bien dans leur composition (effets d'échos, de répétition, de correspondance) que dans le détail de l'écriture. La présence de figures d'analogie dans Archipel n'a donc rien pour surprendre le lecteur, habitué en particulier à l'usage récurrent de la locution "comme si ». Mais les métaphores in absentia sont ici particulièrement fréquentes, et ce qui accentue leur effet poétique, c'est leur isolement syntaxique et typographique, qui distend les liens entre le comparé et le comparant:

nénuphars cernés de clair s'éparpillant sur le fond d'ardoise dérivant (Simon, 2009 :

11).

Séparé par le blanc de tout contexte immédiat, cet énoncé peut se lire de multiples façons. S'agit-il d'une métaphore désignant les îles dispersées à la surface de la mer, qui seront plus loin qualifiées d' "îles nénuphars" (Simon, 2009: 17) ? Ou s'agit-il d'un énoncé littéral désignant de vrais nénuphars, puisque le narrateur signale la présence, parmi la végétation des marais, d'une «fleur de feu au cœur jaune aux pétales vermillon »? À moins que cette "fleur de feu " soit elle-même métaphorique, comme celles qui désignent la flamme des fusils et des canons (Simon, 2009: 15) ? Toutes ces significations sont possibles et se superposent plutôt qu'elles ne s'excluent, et une telle polysémie contribue elle aussi à donner à ces textes un caractère poétique.

On voit comment la découverte d'une géographie singulière devient une source d'inspiration pour Simon, non seulement parce qu'elle le dépayse et bouleverse sa vision du monde, mais parce qu'elle lui suggère une forme d'écriture nouvelle, qui se rapproche plus nettement de la poésie, notamment par le rôle qu'elle accorde à la spatialisation du texte. Celle-ci confirme l'importance que revêt l'espace dans la poétique de Simon: on sait qu'il aime évoquer le travail de l'écriture à l'aide de métaphores spatiales, comme les célèbres «mots-carrefours », et qu'il lui arrive de visualiser la composition de ses textes par des schémas, parfois directement inspirés d'un modèle géologique ou géographique : ainsi du croquis résumant, dans "La fiction mot à mot ", la structure de La Route des Flandres, «l'ensemble se présentant en somme un peu comme ces coupes de terrain au centre desquels se trouve un puits artésien » (Simon, 2006 : 1199).

Dans le texte que je viens de parcourir rapidement avec les instruments de la géographie, de la géocritique et de la géopoétique, la figure de l'Archipel apparaît elle aussi comme une matrice de l'écriture, un modèle spatial qui informe aussi bien l'imaginaire que le style et la mise en page. C'est me semble-t-il un bel exemple des possibilités qu'offre à la littérature et à la critique littéraire la géographie des îles. 


\section{BIBLIOGRAPHIE}

BARTHES, Roland (1972). «Y a-t-il une écriture poétique ?». Le Degré zéro de l'écriture [1953]. Paris : Éditions du Seuil, « Points ».

BENVÉNISTE, Émile (1966). « La phrase nominale ». Problèmes de linguistique générale. Paris : Gallimard.

BRAUDEL, Fernand (1997). «Géohistoire : la société, l'espace, le temps ». Les Ambitions de l'Histoire. Paris : De Fallois, « Le Livre de Poche références ».

CASTIN, Nicolas (1998). Sens et sensible dans la poésie moderne. Paris : PUF.

CERTEAU, Michel de (1990). « Récits d'espace ». L'Invention du quotidien. tome I. Paris : Gallimard, « Folio Essais ».

COLlot, Michel (1997). « La syntaxe nominale ». La Matière-émotion. Paris : PUF, pp. 282-285.

COLLOT, Michel (2005). Paysage et poésie. Paris : Corti.

ColLot, Michel (2014), Pour une géographie littéraire. Paris : Corti.

FRANK, Joseph (1976). « La forme spatiale dans la littérature moderne » (1945), Poétique, n 10, p. 244-266.

HAMON, Philippe (1990). «L'œuvre poétique », in Le Grand atlas des littératures, Encyclopaedia universalis, pp. 44-45.

LAURICHESSE, Jean-Yves (dir.) (2013). Claude Simon géographe. Paris : Classiques Garnier.

MESCHONNIC, Henri (1972). Dédicaces proverbes. Paris : Gallimard.

RICARDOU, Jean (1973). Le Nouveau roman. Paris : Éditions du Seuil.

SIMON, Claude (1969). La Bataille de Pharsale. Paris : Les Éditions de Minuit.

SIMON, Claude (1980). « Roman, description et action », in Paul Hallberg (dir.). The Feeling for nature and the landscape of man. Göteborg: Rundquists Boktryckeri.

SIMON, Claude (1981). Les Géorgiques. Paris : Les Éditions de Minuit.

SIMON, Claude (1987). L'Invitation. Paris : Les Éditions de Minuit.

SIMON, Claude (2006). Euvres, éd. A. Duncan, Paris : Gallimard, « Bibliothèque de la Pléiade ». SIMON, Claude (2009). Archipel et Nord. Paris : Les Éditions de Minuit.

TADIÉ, Jean-Yves (1978). Le Récit poétique. Paris : PUF.

WESTPHAL, Bertrand (2007). La Géocritique : réel, fiction, espace. Paris : Les Éditions de Minuit. WHITE, Kenneth (1994). Le Plateau de l'albatros. Introduction à la géopoétique. Paris : Grasset. ZEMMOUR, David (2008). Une syntaxe du sensible : Claude Simon et la perception. Paris : Presses de l'Université de Paris-Sorbonne. 


\section{NOTES}

1. Archipel a d'abord paru au Danemark en anglais, suédois, finnois et allemand dans Åland 74, Anders Nyborg, Rungsted Kyst, octobre 1973, p.19-24 ; Nord, accompagné de traductions en anglais et en allemand, chez le même éditeur, dans Finland 74, décembre 1973, p. 51-65.

2. J'entends ce terme dans un sens différent de celui que lui donne un de ses inventeurs, et son principal promoteur, Kenneth White (voir White, 1994).

\section{RÉSUMÉS}

Depuis une vingtaine d'années de nombreux travaux ont été consacrés aux liens entre littérature et géographie et l'on a vu émerger de nouvelles approches des textes, baptisées géocritique ou géopoétique. Pour illustrer leur fécondité, Michel Collot propose ici une lecture d'un texte de Claude Simon sur un Archipel décrit avec exactitude mais transfiguré par son imaginaire et son écriture. En associant géographie, géocritique et géopoétique, Michel Collot en dégage successivement la précision documentaire, les valeurs symboliques et la dimension poétique.

Since about twenty years numerous works were dedicated to the links between literature and geography and we saw emerging new approaches of texts, baptized geocritics or geopoetics. To illustrate their fertility, Michel Collot proposes here a reading of Claude Simon's text about an Archipelago described with exactitude but transfigured by his imagination and his way of writing. By associating geography, geocritics or geopoetics, Michel Collot successively clears its documentary precision, symbolic values and poetic dimension.

\section{INDEX}

Keywords : Simon (Claude), Archipelago, geography, geocritics, geopoetics

Mots-clés : Simon (Claude), Archipel, géographie, géocritique, géopoétique

\section{AUTEUR}

\section{MICHEL COLLOT}

Institut Universitaire de France - Université Sorbonne nouvelle - Paris 3 